\title{
Location of the $\beta 4$ Transmembrane Helices in the BK Potassium Channel
}

\author{
Roland S. Wu, ${ }^{1}$ Neelesh Chudasama, ${ }^{1}$ Sergey I. Zakharov, ${ }^{1}$ Darshan Doshi, ${ }^{1}$ Howard Motoike,,${ }^{5}$ Guoxia Liu, ${ }^{1}$ \\ Yongneng Yao, ${ }^{3}$ Xiaowei Niu, ${ }^{1,3}$ Shi-Xian Deng, ${ }^{2}$ Donald W. Landry, ${ }^{2}$ Arthur Karlin, ${ }^{3}$ and Steven 0. Marx ${ }^{1,4}$ \\ Divisions of ${ }^{1}$ Cardiology and ${ }^{2}$ Experimental Therapeutics, Department of Medicine, ${ }^{3}$ Center for Molecular Recognition, Departments of Biochemistry, \\ Physiology, and Neurology, and ${ }^{4}$ Department of Pharmacology, College of Physicians and Surgeons, Columbia University, New York, New York 10032, and \\ ${ }^{5}$ Department of Natural and Applied Sciences, LaGuardia Community College, Long Island City, New York 11101
}

Large-conductance, voltage- and $\mathrm{Ca}^{2+}$-gated potassium $(\mathrm{BK})$ channels control excitability in a number of cell types. BK channels are composed of $\alpha$ subunits, which contain the voltage-sensor domains and the $\mathrm{Ca}^{2+}$ - sensor domains and form the pore, and often one of four types of $\beta$ subunits, which modulate the channel in a cell-specific manner. $\beta 4$ is expressed in neurons throughout the brain. Deletion of $\beta 4$ in mice causes temporal lobe epilepsy. Compared with channels composed of $\alpha$ alone, channels composed of $\alpha$ and $\beta 4$ activate and deactivate more slowly. We inferred the locations of the two $\beta 4$ transmembrane (TM) helices TM1 and TM2 relative to the seven $\alpha$ TM helices, S0 -S6, from the extent of disulfide bond formation between cysteines substituted in the extracellular flanks of these TM helices. We found that $\beta 4$ TM2 is close to $\alpha \mathrm{S} 0$ and that $\beta 4$ TM1 is close to both $\alpha \mathrm{S} 1$ and S2. At least at their extracellular ends, TM1 and TM2 are not close to S3-S6. In six of eight of the most highly crosslinked cysteine pairs, four crosslinks from TM2 to S0 and one each from TM1 to S1 and S2 had small effects on the $V_{50}$ and on the rates of activation and deactivation. That disulfide crosslinking caused only small functional perturbations is consistent with the proximity of the extracellular ends of TM2 to S0 and of TM1 to S1 and to S2, in both the open and closed states.

\section{Introduction}

The large-conductance, voltage- and $\mathrm{Ca}^{2+}$-activated $\mathrm{K}^{+}(\mathrm{BK})$ channels are regulators of cellular excitability in neurons and smooth muscle. $\mathrm{K}^{+}$current through BK channels shifts the membrane potential in the hyperpolarizing direction, suppressing the activity of voltage-dependent $\mathrm{Ca}^{2+}$ channels and thereby decreasing cytoplasmic $\left[\mathrm{Ca}^{2+}\right]$. Both membrane potential and cytoplasmic $\mathrm{Ca}^{2+}$ determine the equilibrium between the closed and the open states (Cui and Aldrich, 2000; Zhang et al., 2001).

The BK $\alpha$ subunit contains a voltage-sensor domain. In addition, the cytoplasmic C-terminal two-thirds of $\alpha$ contains multiple $\mathrm{Ca}^{2+}$-binding sites, including two RCK domains, resembling those of the bacterial $\mathrm{Ca}^{2+}$-gated $\mathrm{K}^{+}$channel MthK (Jiang et al., 2002). The $\mathrm{K}^{+}$conducting pathway is formed by a tetramer of $\alpha$ subunits.

In many cell types, the BK complex contains, in addition, four

\footnotetext{
Received Dec. 30, 2008; revised April 15, 2009; accepted April 25, 2009.

This work was supported in part by National Institutes of Health (NIH) Research Grant Awards P01 HL081172 and R01 HL68093 from National Heart, Lung, and Blood Institute (NHLBI), R01 NS054946 from National Institute of Neurological Disorders and Stroke, and the Arlene and Arnold Goldstein Family Foundation. S.0.M. is an Established Investigator of the American Heart Association. R.S.W. is supported by a Glorney-Raisbeck Fellowship from the New York Academy of Medicine and T32 HL07854 from NIH-NHLBI. D.D. was supported by American Heart Association Founders Affiliate Medical Student Fellowship, and N.C. was supported by the Doris Duke Clinical Research Fellowship Program.

Correspondence should be addressed to either of the following: Steven 0. Marx, Department of Medicine, College of Physicians and Surgeons, 630 West 168th Street, Columbia University, New York, NY 10032, E-mail: sm460@columbia.edu; or Arthur Karlin, Center for Molecular Recognition, College of Physicians and Surgeons, 630 West 168th Street, Columbia University, New York, NY 10032, E-mail: ak12@columbia.edu.

DOI:10.1523/JNEUROSCI.6191-08.2009

Copyright $\odot 2009$ Society for Neuroscience $\quad$ 0270-6474/09/298321-08\$15.00/0
}

$\beta$ subunits. There are four types of $\beta$ subunits, $\beta 1-\beta 4$, the products of the KCNMB1, KCNMB2, KCNMB3, and KCNMB4 genes. Their expression is cell specific (Orio et al., 2002). $\beta 4$ is expressed exclusively in brain. $\beta 4$, like $\beta 1$, slows both activation and deactivation of BK channels. In $\beta 4$ knock-out mice, abnormal BK channel gating kinetics lead to abnormal firing and temporal lobe seizures (Brenner et al., 2005). In the presence of $\mathrm{Ca}^{2+}, \beta 4$, however, has considerably smaller effects than $\beta 1$ on the conductance-voltage relationship (Behrens et al., 2000; Brenner et al., 2000; Meera et al., 2000; Wang et al., 2006). In addition, BK channels with $\beta 4$ are particularly resistant to block by charybdotoxin and iberiotoxin (Behrens et al., 2000; Meera et al., 2000), likely attributable to the large, extracellular loop of $\beta 4$ reaching to and occluding the toxin binding sites in the region of the pore.

The differences in the effects of $\beta 1$ and $\beta 4$ on channel function must reflect differences in their homologous structures and in their interfaces with $\alpha$. We determined previously the proximities and locations of the extracellular ends of the two transmembrane (TM) helices, TM1 and TM2, of $\beta 1$ relative to the seven TM helices, S0-S6, of $\alpha$ (Liu et al., 2008b). We now find that, as in $\beta 1$, $\beta 4$ TM1 is closest to $\alpha \mathrm{S} 1$ and S2 and that $\beta 4$ TM 2 is closest to S0. There are differences in the crosslinking patterns of $\beta 4$ that lead us to consider other arrangements of TM1, TM2 and S0 relative to S1-S6, consistent with the crosslinking data.

\section{Materials and Methods}

Constructs. Mutants of the BK $\alpha$ subunit (mSlo1, KCNMA1; GenBank accession number NM_010610; 1169 residues; molecular weight 131,700 ) and human BK $\beta 4$ subunit (KCNMB4, Open Biosystems clone/ 
accession number 3873518, catalog \#MHS1010-9204471; 210 residues; molecular weight 23,800) were generated by site-directed mutagenesis using the QuikChange XL (Stratagene) kit. Cys substitutions in $\alpha$ (Fig. $1 A$ ) were made in a pseudo-wild-type (pWT) background, in which Cys14 and Cys141 were each mutated to Ala. The $\mathrm{C}$ terminus of the $\beta 4$ construct was tagged with a $3 \mathrm{X}$ FLAG epitope (DYKDHDGDYKDHDIDYKDDDDK). Cys substitutions in $\beta 4$ (Fig. $1 B$ ) were made in a pWT background, in which Cys 38 and Cys 173 were each mutated to Ala.

Expression, surface biotinylation, and cell lysis. HEK293 cells were cultured and transfected as described previously (Morrow et al., 2006). After $24-48 \mathrm{~h}$, the cells were collected, washed, and biotinylated with $2 \mathrm{~mm}$ sulfosuccinimidyl6-(biotinamido) hexanoate (sulfoNHS-LCbiotin) (Pierce), a membrane-impermeant reagent, in Dulbecco's PBS (DPBS). The cells were solubilized for $20 \mathrm{~min}$ at $4^{\circ} \mathrm{C}$ in lysis buffer

(1\% Triton X-100, $150 \mathrm{~mm} \mathrm{NaCl}, 50 \mathrm{~mm}$ Tris, 1 mм EDTA, 2 mм $\mathrm{N}$-ethylmaleimide, and Roche complete protease inhibitors). Insoluble material was removed by centrifugation.

Treatment with dithiothreitol and QPD. HEK293 cells were kept on the tissue culture dish, washed, and biotinylated with $4 \mathrm{~mm}$ sulfoNHS-LCbiotin solution. The cells were then washed with DPBS, and a solution of $137 \mathrm{~mm} \mathrm{NaCl}, 2.7 \mathrm{~mm} \mathrm{KCl}, 0.1 \mathrm{~mm} \mathrm{CaCl}_{2}, 0.1 \mathrm{~mm} \mathrm{MgCl}_{2}$, and $40 \mathrm{~mm}$ HEPES was added to the dish. Dithiothreitol (DTT) was added for a final concentration of $20 \mathrm{~mm}$. These cells were then washed with DPBS, collected, and lysed as detailed above.

In most experiments, no oxidizing agent was added to the cells. In experiments to test the susceptibility of two Cys on the cell surface to reform a disulfide after reduction, as described previously (Liu et al., 2008a), we used the doubly charged oxidant, $4,4^{\prime}$-(azodicarbonyl)-bis[1,1-dimethylpiperazinium, diiodide] [quaternary piperazinium diamide (QPD)] (Kosower et al., 1974). For cells treated with QPD, cells were resuspended in a solution of $137 \mathrm{~mm} \mathrm{NaCl}, 2.7 \mathrm{~mm} \mathrm{KCl}, 0.9 \mathrm{~mm}$ $\mathrm{CaCl}_{2}$, and $0.49 \mathrm{mM} \mathrm{MgCl}_{2}$. QPD was then added to a final concentration of $40 \mu \mathrm{M}$. Cells were then washed and lysed as detailed above.

Quantitating crosslinking. The cell lysate was mixed with NeutravidinSepharose beads (Pierce) for $90 \mathrm{~min}$ at room temperature. In a typical sample, lysate of cells from a $60 \mathrm{~mm}$ plate in $300 \mu \mathrm{l}$ of lysis buffer was mixed with $30 \mu \mathrm{l}$ of a $50 \%$ slurry of Neutravidin beads, previously washed and suspended in lysis buffer. The beads were washed in a solution of $0.05 \%$ SDS, $40 \mathrm{~mm}$ HEPES, pH 7.4, and 1 mM EDTA. To elute bound protein, we added $80 \mu \mathrm{l}$ of $6 \mathrm{~m}$ urea, $4 \%$ SDS, $200 \mathrm{~mm}$ Tris, $\mathrm{pH} 8$, and $2 \mathrm{~mm}$ EDTA and heated the mixture to $100^{\circ} \mathrm{C}$ for $2 \mathrm{~min}$. After a short spin, the supernatant was collected. The samples were then electrophoresed, transferred to nitrocellulose, and immunoblotted by sequential treatment with anti-BK- $\alpha$-C-terminus antibody (BD Biosciences) or anti-FLAG antibody (Sigma-Aldrich), horseradish-peroxidaseconjugated secondary antibody, and ECL reagent (Pierce Super Signal West). We quantitated the chemiluminescence from each band with a CCD camera (Carestream Health) and ImageQuant software (Molecular Dynamics). We calculated the extent of crosslinking, from the band at $155 \mathrm{kDa}$ divided by the sum of the bands at 125 and $155 \mathrm{kDa}$. All mutants were independently expressed and tested twice if there was little crosslinking and three or more times if there was substantial crosslinking. In each experiment, duplicate lanes were run. Finally, the averages of the individual means were calculated. Intrasubunit crosslinking of $\alpha$, with or without the presence of $\beta 4$, was determined as described previously (Liu et al., 2008a).

For comparing the crosslinking of different pairs of flanks, we calculated a weighted mean of the top three extents of crosslinking between these flanks. We weighted the individual Cys pairs in the two flanks by the sum of the number of residues that the two Cys were out from the membrane, for example, if one Cys was the second residue out from the membrane in the first flank and the other Cys was the third residue out from the membrane in the second flank, the extent of crosslinking was multiplied by $1 / 5$. The weighted mean was the sum of the weighted extents of crosslinking divided by the sum of the weights.

Functional effects of crosslinking. Conductance-voltage ( $G-V)$ curves and the activation and deactivation kinetics were determined as described previously (Zakharov et al., 2005; Morrow et al., 2006; Liu et al., $2008 \mathrm{~b})$. We determined the effects of mutation and crosslinking on $V_{50}$ and on the rate constants of activation $\left(k_{\text {act }}\right)$ and deactivation $\left(k_{\text {deact }}\right)$. The effect on $V_{50}$ was calculated as $\Delta V_{50}=V_{50, \mathrm{MUT}}-V_{50, \mathrm{pWT}}$. The effects on the rate constants of opening and closing were calculated as $\log \left(k_{\mathrm{MUT}} / k_{\mathrm{pWT}}\right)$. We calculated the effect attributable to substituting the disulfide-bonded cysteine (i.e., completely crosslinked Cys) and the effect attributable to substituting two reduced cysteines (i.e., completely reduced Cys) as described previously (Liu et al., 2008b).

\section{Results}

\section{Mutant $\alpha$ and $\beta 4$}

We mutated to Cys, one at a time, at least the first four residues predicted to be just extracellular to the membrane-embedded residues of $\alpha$ S0-S6 and similarly of $\beta 4$ TM1 and TM2 (Fig. $1 A, B)$. The mutations were made in pWT $\alpha$ and $\beta 4$ constructs (see Materials and Methods). We showed previously that the functional characteristics ( $V_{50}$ and rate constants for activation and deactivation) of pWT $\alpha$ are only slightly different from those of WT $\alpha$ (Liu et al., 2008a). We now have found that the functional properties of channels formed by pWT $\alpha$ and pWT $\beta 4$ differ only slightly from those of wild-type $\alpha$ and $\beta 4$ (data not shown). All 208 combinations of 26 Cys-substituted mutants of pWT $\alpha$ and eight Cys-substituted mutants of pWT $\beta 4$ were coexpressed in HEK293 cells. The proteins on the cell surface of the intact cells were labeled with an impermeant biotinylation reagent. The detergent-solubilized, biotinylated cell-surface proteins were captured on Neutravidin beads and released in nonreducing SDS-PAGE sample buffer.

We found that almost all combinations of a Cys-substituted $\alpha$ and a Cys-substituted $\beta 4$ were well expressed on the cell surface. Only combinations $\alpha$ A295C and $\alpha$ T297C expressed poorly and were not further analyzed.

Disulfide crosslinking of Cys-substituted $\alpha$ and $\beta 4$ mutants As described previously (Liu et al., 2008a,b), we determined the extent of disulfide bond formation in surface-expressed channels in the absence of any oxidizing agent other than ambient oxygen. This endogenous disulfide bond formation could be attributable 
A

\begin{tabular}{|c|c|c|c|c|c|c|c|c|c|c|c|}
\hline \multirow[b]{2}{*}{$\alpha$} & \multirow[b]{2}{*}{ pWT } & \multirow[b]{2}{*}{ pWT } & \multicolumn{4}{|c|}{ S0-TM2 } & \multicolumn{2}{|c|}{ S1-TM1 } & \multicolumn{2}{|c|}{ S2-TM1 } & \\
\hline & & & $\mathrm{R} 17 \mathrm{C}$ & G18C & Q19C & $\mathrm{R} 20 \mathrm{C}$ & $\mathrm{N} 136 \mathrm{C}$ & P137C & F144C & D147C & \\
\hline$\beta$ & pWT & I168C & I168C & I168C & I168C & I168C & $\mathrm{A} 43 \mathrm{C}$ & S41C & L44C & L44C & \\
\hline$\overline{\text { DTT }}$ & $-\quad+$ & $-\quad+$ & $-\quad+$ & $-\quad+$ & $-\quad+$ & $-\quad+$ & $-\quad+$ & $-\quad+$ & $-\quad+$ & $-\quad+$ & \\
\hline $\begin{array}{l}\mathrm{kDa} \\
150 \rightarrow\end{array}$ & -- & -- & $=$ & - & - & $=-$ & e & $=$ & $=$ & $=$ & $\stackrel{\leftarrow \alpha-\beta}{\leftarrow \alpha}$ \\
\hline & 0 & 0 & .85 & .90 & .85 & .88 & .89 & .87 & .93 & .85 & \\
\hline
\end{tabular}

B

\begin{tabular}{|c|c|c|c|c|c|c|c|c|c|c|c|c|c|}
\hline$\alpha$ & \multicolumn{3}{|c|}{ pWT } & \multicolumn{3}{|c|}{ So-TM2 } & \multicolumn{3}{|c|}{ S1-TM1 } & \multicolumn{3}{|c|}{ S2-TM1 } & \\
\hline$\beta$ & & WT & & & 1680 & & & 410 & & & $44 C$ & & \\
\hline$\overline{\text { DTT }}$ & - & + & + & - & + & + & - & + & + & - & + & + & \\
\hline QPD & - & - & + & - & - & + & - & - & + & - & - & + & \\
\hline $\begin{array}{l}\mathrm{kDa} \\
150 \rightarrow\end{array}$ & & & & $=$ & & $=$ & $=$ & - & $=$ & $=$ & & $=$ & $\leftarrow \alpha-\beta$ \\
\hline $100 \rightarrow$ & $\overline{0}$ & $\frac{-}{0}$ & 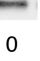 & .87 & 0 & .59 & & .19 & .84 & & .06 & .69 & \\
\hline
\end{tabular}

Figure 2. Detecting disulfide crosslinking between $B K \alpha$ and $\beta 4$ subunits. $A$, Western blots illustrating endogenous crosslinking of Cys-substituted $\alpha$ and Cys-substituted $\beta 4$. Expression of mutants, selection of channels transported to the cell surface, SDS gel electrophoresis, and blotting are described in Materials and Methods. The blots were developed with an antibody against an epitope from the $($ terminus of $\alpha$, which recognizes $\alpha(125 \mathrm{kDa}$ ) and the crosslinked $\alpha-\beta 4$ complex ( $\sim 155 \mathrm{kDa}$ ). Half of each sample was reduced with DTT (+DTT lanes). Under each gel is the fraction of crosslinked $\alpha-\beta 4$ complex in the unreduced lane. $B$, Reduction and reoxidation of the disulfide between R17C and I168C, P137C and S41C, and F144C and L44C. Channels on intact cells were reduced with DTT and oxidized for $20 \mathrm{~min}$ with $40 \mu \mathrm{m}$ QPD. At the bottom of each lane is the fraction of crosslinked $\alpha-\beta 4$ complex.

to spontaneous oxidation by dissolved oxygen or to catalytic oxidation by protein disulfide isomerase (PDI) homologs in the endoplasmic reticulum (Wilkinson and Gilbert, 2004) or by secreted PDI homologs (Jiang et al., 1999) or secreted oxidases (Becker et al., 2006). Whatever the mechanism of disulfide bond formation, we assume that the extent of formation reflects the relative proximity of the two Cys before disulfide bond formation.

The mobility of pWT $\alpha$ by SDS-PAGE corresponds to an apparent molecular mass of $\sim 125 \mathrm{kDa}$ (Fig. $2 \mathrm{~A}$ ). Coexpressed with pWT $\alpha$, pWT $\beta 4$ ran as three bands in the range of $25-35 \mathrm{kDa}$ (supplemental Fig. 1, available at www.jneurosci.org as supplemental material). These bands correspond to an unglycosylated, a core glycosylated, and a maturely glycosylated $\beta 4$ (Jin et al., 2002). The disulfide crosslinked dimer of Cys-substituted $\alpha$ and Cys-substituted $\beta 4$ ran as one or two very close bands of apparent molecular mass $\sim 155 \mathrm{kDa}$ (Fig. 2). Several examples of pairs of Cys-substituted $\alpha$ and $\beta 4$ that gave this crosslinked complex are shown. The $\sim 155 \mathrm{kDa}$ band was absent in extracts of cells expressing pWT $\alpha$ and pWT $\beta 4$ (Fig. 2) or from cells coexpressing Cys mutants of $\alpha$ and pWT $\beta 4$ or, conversely, of pWT $\alpha$ and Cys mutants of $\beta 4$ (data not shown); no single mutant yielded the $\sim 155 \mathrm{kDa}$ band. Furthermore, the $\sim 155 \mathrm{kDa}$ band contained $\alpha$ crosslinked to $\beta 4$ : the band disappears after reduction of the sample with DTT, and the $125 \mathrm{kDa} \alpha$ band appears (Fig. $2 \mathrm{~A}, \mathrm{~B}$ ). In addition, the $\sim 155 \mathrm{kDa}$ band, but not the $125 \mathrm{kDa}$ band, bound an anti-FLAG antibody that recognized the FLAG-tagged $\beta 4$ (supplemental Fig. 2, available at www.jneurosci.org as supplemental material).

The disulfide bond between Cys-substituted $\alpha$ and $\beta 4$ could be reduced in intact cells by DTT and reoxidized on the cell surface with the membrane-impermeant, quaternary ammonium diamide derivative QPD. This is shown for three pairs of Cys-substituted $\alpha$ and $\beta 4$ that were initially almost completely endogenously crosslinked: one pair each from $\alpha$ S0 $-\beta 4$ TM2, $\alpha$ S1- $\beta 4$ TM1, and $\alpha$ S2- $\beta 4$ TM1 (Fig. $2 B$ ).

\section{Quantitating $\boldsymbol{\alpha}-\boldsymbol{\beta} 4$ subunit crosslinks} Cys substituted in the TM1 flank formed disulfides most readily with Cys in the flanks of S1 and S2 (Fig. 3A), whereas Cys in the flank of TM2 formed disulfides most readily with Cys in the flank of S0 (Fig. 3B). Among the 16 combinations of a Cys in the TM1 flank and a Cys in the S1 flank, seven combinations formed disulfides to an extent $>60 \%$ (Fig. $3 A$ ). The average of the top three extents of crosslinking was $77 \pm$ $4 \%$. Similarly, among the 16 combinations of a Cys in the TM1 flank and a Cys in the S2 flank, nine combinations formed disulfides to an extent $>60 \%$ (Fig. $3 A$ ). The average of the top three extents of crosslinking was $78 \pm 10 \%$. In contrast, the TM1 flank crosslinked much less to the flanks of S0, S5, and S6 or the S3-S4 loop. The number of combinations of a Cys in these other flanks and a Cys in the TM1 flank that formed disulfides to an extent $>20 \%$ was none for S0, three for S3-S4, one for S5, and one for S6. Only one of these combinations, TM1 A43C and S3-S4 N200C, reached $30 \%$ crosslinking. The averages of the top three extents for S3-S4, S5, and S6 were $27 \pm 3,14 \pm 7$, and $14 \pm 13 \%$, respectively. By either criterion, the extracellular flank of TM1 is closer to the flanks of S1 and S2 than to the flanks of S3-S4, S5, and S6.

Disulfide crosslinking from TM2 was even more restricted than from TM1. Among the 16 combinations of a Cys in the TM2 flank and a Cys in the S0 flank, 11 combinations formed disulfides to an extent $>60 \%$ (Fig. $3 B$ ). The average of the top three extents was $81 \pm 5 \%$. There was little disulfide formation with the flanks of S1 and S2 or the S3-S4 loop: the averages of the top three extents were $4 \pm 1,10 \pm 3$, and $7 \pm 1 \%$, respectively. There was $40-50 \%$ crosslinking from two TM2 flanking positions to one position in the S5 flank and no crosslinking to the other three S5 flanking positions. The average of the top three extents of crosslinking was $38 \pm 16 \%$. One Cys in the TM2 flank, V169C, crosslinked just $<30 \%$ to S6 flank K296C. The average of the top three extents was $21 \pm 7 \%$. The flank of TM2 is close to the flank of S0, has some access to the flanks of S5 and S6, and has little access to the flanks of S1 and S2 and the S3-S4 loop.

We have represented the salient features of the crosslinking as lines linking the extracellular ends the TM helices of $\alpha$ and $\beta 4$ drawn as circles (Fig. 4). The circles labeled S1-S6 were superimposed on the extracellular ends of these helices in two structures of $K_{v}$ 1.2. The structure underlying model 1 is a Rosetta model of the closed state (Yarov-Yarovoy et al., 2006), and the structure underlying models 2 and 3 is the $\mathrm{x}$-ray crystallographic solution of the presumed open state of the potassium channel $\mathrm{K}_{\mathrm{v}} 1.2$ (Long et al., 2005). In models 1 and 2, the extracellular end of S0 is in a cove between S2 and S3, in which we placed it previously (Liu et al., 2008a). In model 3, the extracellular end of S0 is placed outside of the voltage-sensor domain helices S1-S4, close to the short loop between S3 and S4. In each of the models, TM1 is placed close to $\mathrm{S} 1$ and $\mathrm{S} 2$, and TM2 is placed close to S0, as is consistent with 
the crosslinking results. It is important to note that the crosslinking of S0 to S1 and to S3-S4 was not different in the presence of $\beta 4$ (supplemental Fig. 3, available at www. jneurosci.org as supplemental material) than it was in the presence of $\beta 1$ or in the absence of either $\beta$ (Liu et al., 2008a).

The mean of the top three extents of crosslinking for each pair of flanks were calculated as above, except that the extents were weighted by the inverse of the sum of the number of residues the Cys were away from the membrane, giving greater weight to crosslinks that were close to the membrane than to those that were farther away. These means were binned, and the three bins were represented by lines of different thicknesses (Fig. 4). In addition, to represent the distance of the crosslinks from the membrane, the lines were color coded in bins of the mean sum of the number of residues the Cys were away from the membrane. As we showed previously, close to the membrane, the extracellular end of S0 is next to the short loop between S3 and S4 (Liu et al., 2008a). The $\beta 4$ helix TM1 is closest to $\mathrm{S} 1$ and S2, and TM2 is closest to S0, although these crosslinks are on average farther from the membrane than the crosslinks between $\mathrm{S} 0$ and S3-S4.

Functional effects of the crosslinks Three functional parameters were determined: $V_{50}$ for activation and the rate constants for activation and deactivation (Fig. $5)$. The effect on $V_{50}$ was taken as $V_{50}$ for the channel composed of Cys-substituted $\alpha$ and Cys-substituted $\beta 4$ minus $V_{50}$ for the channel composed of pWT $\alpha$ and pWT $\beta 4$. The effect on rate constants was taken as the log of the quotient of the rate constant for the mutant channel divided by the rate constant for pWT channel. For each channel, we characterized its function both before reduction with DTT, when $\alpha$ and $\beta 4$ were predominantly disulfide crosslinked, and after reduction with DTT, when they were predominantly reduced. Using the densities of the $\alpha$ band and the $\alpha-\beta 4$ band in the immunoblots from the cells before and after reduction with DTT, under the same conditions used in the functional studies (supplemental Table 1, available at www.jneurosci.org as supplemental material), we estimated the fraction of disulfidecrosslinked dimer and of reduced monomer under the two conditions. We assumed that the extent of each functional effect was the linear sum of the effect of the disulfide times the extent of crosslinking plus the effect of the two reduced Cys times the extent of reduction. From two equations, one for the oxidized state and one for the reduced state, in two unknowns, we calculated the functional effect attributable to the two Cys substitu-
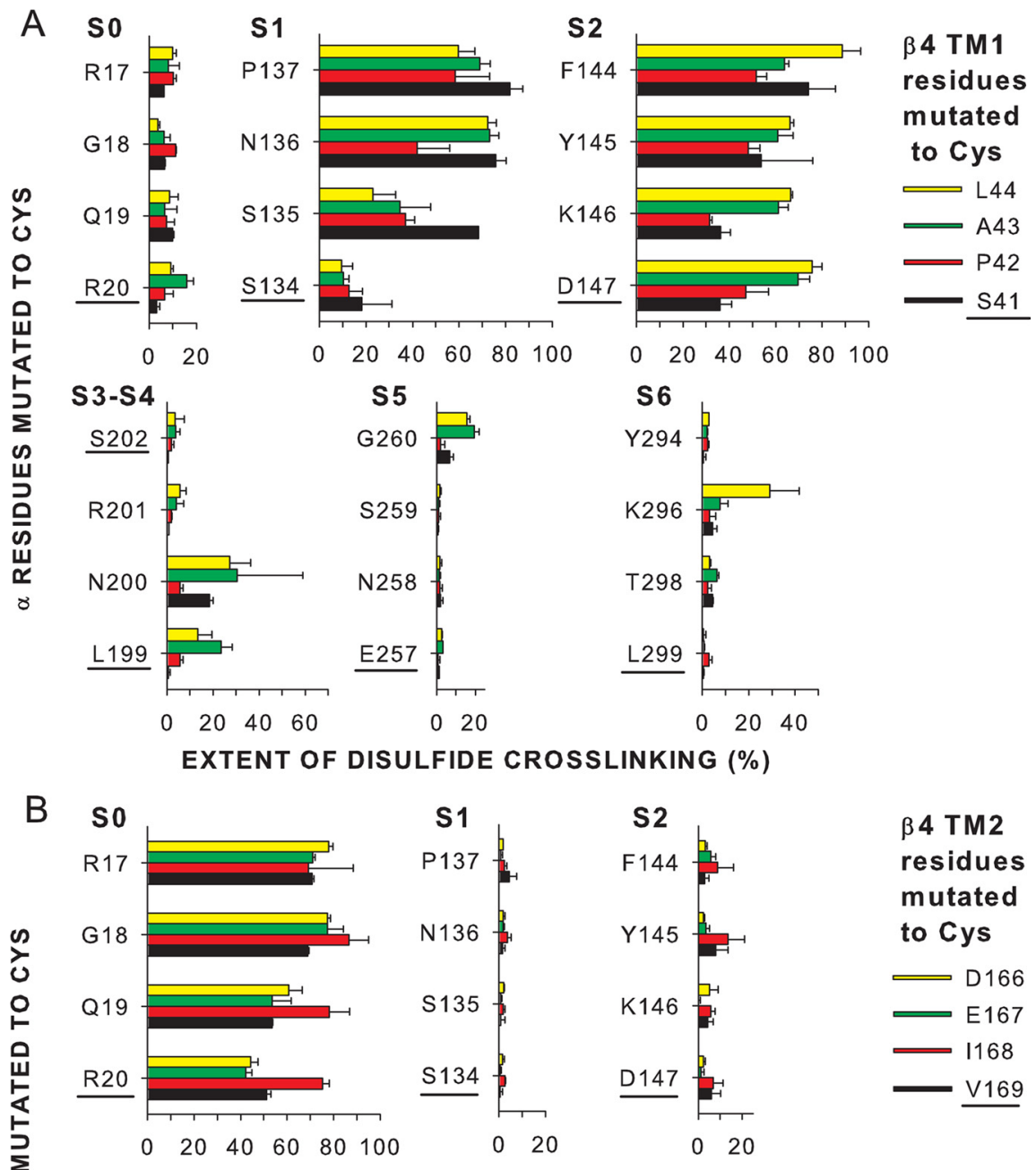

Figure 3. Extents of endogenous disulfide bond formation between Cys substituted in the extracellular flanks of $\alpha$ and $\beta 4$. $A$, $\alpha$ SO -S6 flanks and $\beta 4$ TM1 flank. $\boldsymbol{B}, \alpha$ S0 -S6 flanks and $\beta 4$ TM2 flank. The $\alpha$ residues substituted by Cys are indicated along the left edge of each vertical axis, and the $\beta 4$ residues substituted by Cys are color coded as shown. The extent of disulfide bond formation is represented by bars in the horizontal direction. In the cases in which the mean extent of disulfide bond formation was zero, the value $0.5 \%$ was plotted to identify these pairs as tested. The residues closest to the membrane are underlined.

tions (i.e., completely reduced state) and the functional effect attributable to the disulfide (i.e., completely oxidized state) (Liu et al., 2008b). The functional parameters of the channel composed of pWT $\alpha$ and pWT $\beta 4$ was unaffected by DTT (Fig. $5 B$ ).

We determined the functional properties of eight combinations of Cys-substituted $\alpha$ and Cys-substituted $\beta 4$. Four involved S0 and TM2, two involved S1 and TM1, and two involved S2 and TM1. All eight double-Cys mutant channels formed disulfides to an extent $65 \%$ or greater. All were functional in both the fully reduced and fully oxidized forms: they 

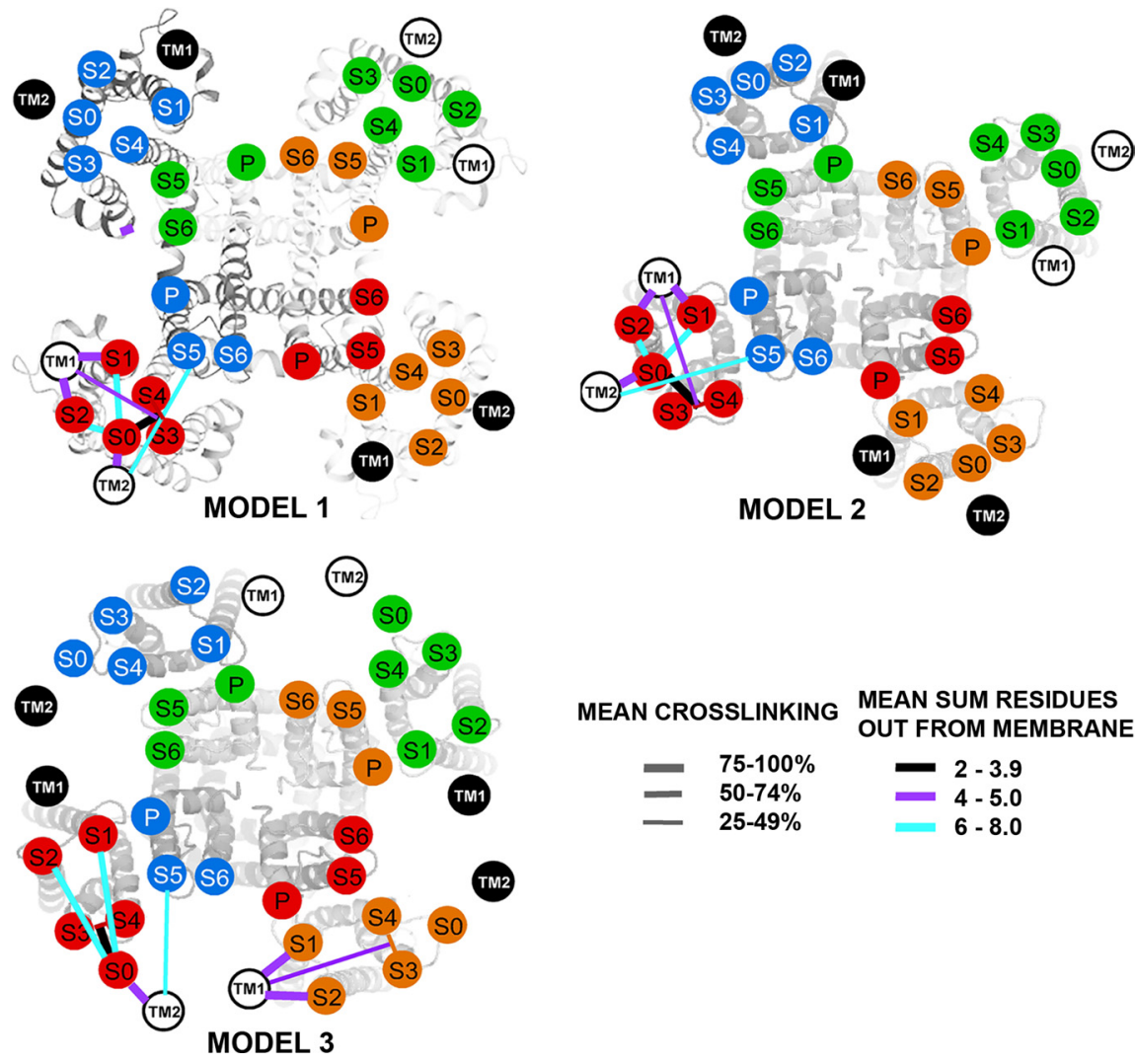

Figure 4. Locations of the extracellular ends of $\beta 4 \mathrm{TM} 1$ and TM2 relative to the extracellular ends of $\alpha \mathrm{S} 0-\mathrm{S} 6$. Model 1, A Rosetta model of $\mathrm{K}_{\mathrm{v}} 1.2$ in the closed state (Yarov-Yarovoy et al., 2006) is the template for locating the extracellular ends of BK $\alpha$

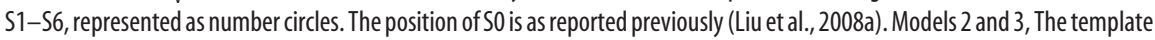
is the crystal structure of $\mathrm{K}_{v} 1.2$ in the open state (Long et al., 2005). In Model 2, the position of S0 relative to $\mathrm{S1}-\mathrm{S} 4$ is the same as in model 1. In model 3, the position of $\mathrm{SO}$ is outside of $\mathrm{S1}-\mathrm{S4}$. The locations of the extracellular ends of $\beta 4 \mathrm{TM} 1$ and TM2 are represented by labeled black and white circles. TM1 and TM2 from two $\beta 4$ subunits are represented by white circles with black numbers and two $\beta 4$ subunits by black circles with white letters. In models 1 and 2 , TM1 and TM2 from one $\beta 4$ subunit straddle one $\alpha$ subunit. In model 3, TM1 and TM2 from one $\beta 4$ subunit are between two $\alpha$ subunits. For each pair of flanks, the mean of the top three extents of crosslinking (see Materials and Methods) is coded by the thickness of the connecting line (see legend in figure). To indicate the distance of a crosslink from the ends of the TM helices, we added the number of residues out from the membrane of each of the two Cys. The mean of these distances for the top three extents of crosslinking is indicated by the color of the line (see legend in figure). P, Pore.

were activated by depolarization and deactivated by hyperpolarization. Therefore, none of the residues mutated to Cys was irreplaceable for function.

In six of the eight pairs of mutants tested, the disulfide changed $V_{50}<16 \mathrm{mV}$ in either the positive or negative direction (Fig. 6A). The six included the four pairs involving the S0 flank and the TM2 flank, S1 N136C and TM1 A43C, and S2 D147C and TM1 L44C. In all of these cases, the effect of substituting the two reduced Cys was equal or greater than the effect of the disulfidecrosslinked Cys (Fig. 5C), although none of the effects were large. Disulfide crosslinking of these six pairs also perturbed the activation rate constant only slightly, within $0.4-2.1$ times (Fig. $6 B$ ). In contrast, crosslinking of the pair S1 P137C and TM1 S41C (Fig. 5D) and the pair S2 F144C and TM1 L44C (Fig. $5 E$ ) increased $V_{50}$ by $\sim 60$ $\mathrm{mV}$, and, also in these two cases, $k_{\text {act }}$ decreased 7- to 8-fold and increased $k_{\text {deact }} 1.5$ - to 2.1 -fold. Considering the effect of crosslinking one flank to another, the average changes in $V_{50}$ attributable to crosslinking TM1 to S1 and TM1 to S2 were greater than that attributable to crosslinking TM2 to S0 (supplemental Fig. 4, available at www.jneurosci.org as supplemental material).

\section{Discussion}

Proximity inferred from disulfide crosslinking

We inferred from the extent of disulfide bond formation between Cys in $\alpha$ and Cys in $\beta 4$ that the extracellular end of TM1 is close to the extracellular ends of S1 and S2 and that the extracellular end of TM2 is close to the extracellular end of S0 (Fig. 4). The disulfide crosslinking that we measured was restricted to channels transported to the cell surface. Because the cell selects against transporting misfolded or misassembled proteins (Nikles and Tampé, 2007; Nakatsukasa and Brodsky, 2008), the structures of these channels are likely to be at least near native. Also, the greater the extent of crosslinking of two Cys, the closer on average they were before crosslinking (Liu et al., 2008a). We tried to average the effects of factors other than proximity by testing multiple pairs of Cys in each pair of flanks.

\section{Model of BK $\alpha$ and $\beta 4$}

Taking three-dimensional structures of $\mathrm{K}_{\mathrm{v}} 1.2$ as templates, we tested which arrangements of S0 and of TM1 and TM2 were consistent with the crosslinking. Previously, we (Liu et al., 2008a) picked the Rosetta-generated model for $\mathrm{K}_{\mathrm{v}} 1.2$ in the closed state (Yarov-Yarovoy et al., 2006) as the template, because it is likely that the crosslinking we observed occurred in the closed state of the BK channel. It is possible, however, that in the closed state of the BK channel, the voltage-sensing domains are close to their position in the open state (Ma et al., 2006). Furthermore, even in the closed state of $\mathrm{K}_{\mathrm{v}} 1.2$, the extracellular end of $\mathrm{S} 1$ is close to the pore helix, as it appears in the in $\mathrm{K}_{\mathrm{v}} 1.2$ crystal structure (Lee et al., 2009). This leads us to consider also the high-resolution structure of the open state of $\mathrm{K}_{\mathrm{v}} 1.2$ (Long et al., 2005) as a template. The important difference between the structures of the closed state and the open state for our purposes is the counterclockwise rotation of the voltage sensor in the modeled closed state relative to the solved open state.

We (Liu et al., 2008a) previously located the extracellular end of S0 between S2 and S3 in the closed state model (Fig. 4, model $1)$. We now also consider the open state with S0 placed between S2 and S3 (model 2) and the open state with S0 placed outside of S3-S4 (model 3). In models 1 and 2, the observed crosslinking of the extracellular flank of S0 to the flanks of S1, S2, and S3-S4 is readily rationalized. This location of S0 in model 1 is also consistent with the absence of crosslinking of S0 to S5 and S6. In model 3 , however, in which S3-S4 is rotated away from S5 and S6, S0 can also be placed outside the S1-S4 bundle, close to S3-S4 without S0 being close to S5 or S6.

Model 3 has the advantage that S0 does not disrupt the S1-S4 bundle; however, S0 is farther from S1 and S2 in model 3 than in models 1 and 2. There was extensive crosslinking from the flank 
A

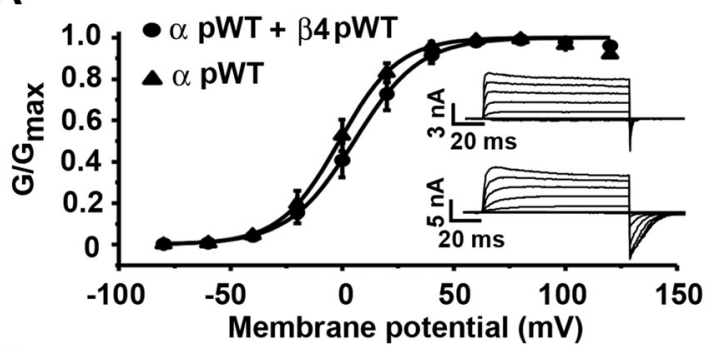

B

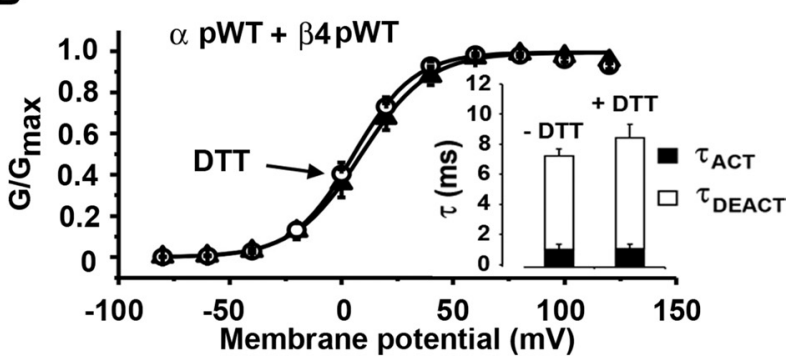

C

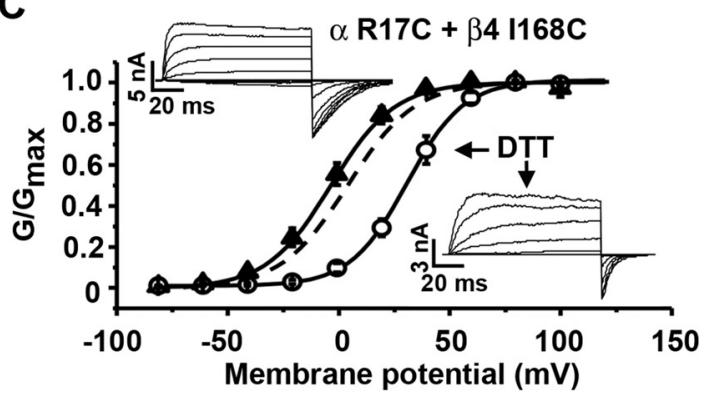

D

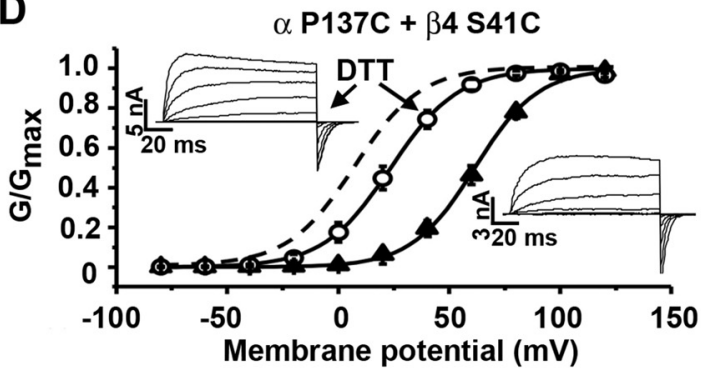

E

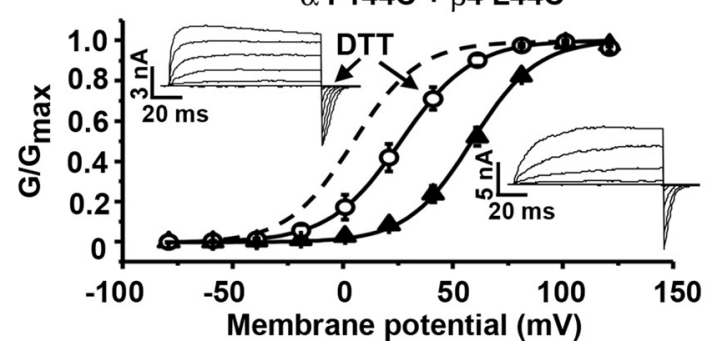

Figure 5. Activation, deactivation, and conductance-voltage curves for pWT and mutant channels. A, Normalized conductance as a function of voltage ( $G-V$ curve) for pWT $\alpha$ (filled triangles) and pWT $\alpha$ and pWT $\beta 4$ (filled circles). The recordings were made in outside-out macropatches with $10 \mu \mathrm{M} \mathrm{Ca}{ }^{2+}$ inside the pipette. Inset, Macroscopic currents conducted by pWT $\alpha$ (top) and pWT $\alpha$ and pWT $\beta 4$ (bottom) in response to step depolarizations from -80 to $+140 \mathrm{mV}$. B, G-V curve for pWT $\alpha+$ pWT $\beta 4$ before (filled triangle) and after $20 \mathrm{~mm}$ DTT (open circle). Inset, Graph showing $\tau_{\text {ACT }}$ and $\tau_{\text {DEACT }}$ in absence and presence of DTT. $\mathbf{C}-\boldsymbol{E}$, Macroscopic currents and $\mathrm{G}-V$ curves were determined with $10 \mu \mathrm{M} \mathrm{Ca}^{2+}$ inside the pipette before (filled triangles) and after (open circles) DTT for $\alpha$ R17C and $\beta 4$ I168C (C), $\alpha$ P137C and $\beta 4 \mathrm{~S} 41 \mathrm{C}(\boldsymbol{D})$, and $\alpha \mathrm{F} 144 \mathrm{C}$ and $\beta 4 \mathrm{~L} 44 \mathrm{C}(\boldsymbol{E})$. Dashed lines represent the $\mathrm{G}-V$ curve for pWT $\alpha$ and pWT $\beta 4$. Error bars are SD. of S0 to the flanks of S1 and S2, but this was predominantly between Cys three or four residues out from the membrane on both flanks. This distance out from the membrane in both $\mathrm{S} 0$ and in S1 or S2 might accommodate the flank of S0 reaching over the loop between S3 and S4 to the flanks of S1 and S2. In the native BK $\alpha$ structure, there is a disulfide bond between Cys14, seven residues out from the membrane domain of S0, and Cys141, eight residues out from both $\mathrm{S} 1$ and S2, in the middle of the loop between them, indicating that this is the direction taken by the SO flank in the native structure.

In all three models, the positions of $\beta 4 \mathrm{TM} 1$ and TM2 are consistent with the high extents of crosslinking of TM2 to $\mathrm{S} 0$ and of TM1 to S1 and S2. The low extents and the absence of crosslinking are also informative. The $25-30 \%$ crosslinking of TM1 to the S3-S4 loop and the absence of TM1 crosslinking to S0 favor model 3 because, if the TM1 flank can crosslink to the S3-S4 loop, it must pass S0 in models 1 and 2 but not in model 3 . Furthermore, the $\sim 40-50 \%$ crosslinking of TM2 to S5, albeit between three and four residues out from the membrane on the TM2 flank and four residues out from the membrane on the S5 flank, and the absence of TM2 crosslinking to S3-S4 favor model 3. A notable difference between the models is that, in models 1 and 2, a $\beta$ subunit associates with a single $\alpha$ voltage-sensor domain, whereas in model 3 , a single $\beta$ associates with the voltagesensor domains of two $\alpha$ subunits.

The loop between $\beta 4$ TM1 and TM 2 reaches the vicinity of the central pore, in which it blocks binding of charybdotoxin and iberiotoxin (Behrens et al., 2000; Meera et al., 2000), so that the flanks of TM1 and TM2, the beginning and end of the loop, are headed in the direction of the extracellular flanks of S5 and S6. In all three models, the loop, containing 130 residues, could form a structure that reaches that far. In model 1 , for example, the distances from the centers of the disks representing TM1 and TM2 are both $\sim 40 \AA$ from the center of the pore, which could be spanned by the 130 residues in the $\beta 4$ loop. As an example, siamensis toxin 3 contains 71 residues and five disulfide bonds and forms a flat structure that spans $\sim 20 \times \sim 40 \AA$ (Betzel et al., 1991).

\section{Comparison with $\beta 1$}

The patterns of crosslinking of $\beta 1$ (Liu et al., 2008b) and of $\beta 4$ (Fig. 3) were somewhat different (supplemental Fig. 4, available at www.jneurosci.org as supplemental material). TM2 of $\beta 1$ was even more extensively crosslinked to S0 than was TM2 of $\beta 4$, and furthermore there was more crosslinking close to the membrane in $\beta 1$ than in $\beta 4$. In $\beta 1$, unlike in $\beta 4$, TM2 crosslinked to $S 2$ to an average extent of $36 \%$ (mean of top three extents). This result looks more likely in models 1 and 2 than in model 3 . The absence of crosslinking of $\beta 4$ TM2 to $\$ 2$ could be a result of the distance in model 3 or the close association of TM1 with S2, blocking access to TM2. Also, in $\beta 1$, the mean extent of crosslinking of TM1 to S0 was $42 \%$, whereas in $\beta 4$, there was no crosslinking of TM1 to S0. Conversely, $\beta 1$ TM1 did not crosslink to S3-S4, whereas $\beta 4$ TM1 did crosslink to S3-S4 to an average extent of 27\%. In model 3, for example, this difference could be attributable to the closer association of $\beta 4$ TM1 than $\beta 1$ TM1 with S1 and S2 and, consequently, the shorter distance of TM1 to S3-S4. Although we show that $\beta 4$ TM2 can crosslink weakly to S5, consistent with model 3, the aligned pair of Cys was not tested in $\beta 1$. The subtle differences between $\beta 1$ and $\beta 4$ crosslinking to $\alpha$ may reflect differences in the positions of TM1 and TM2 relative to S0-S6 or differences in the structures and flexibilities of the two $\beta \mathrm{s}$, in which the extracellular loops have different numbers of residues and of disulfides. 

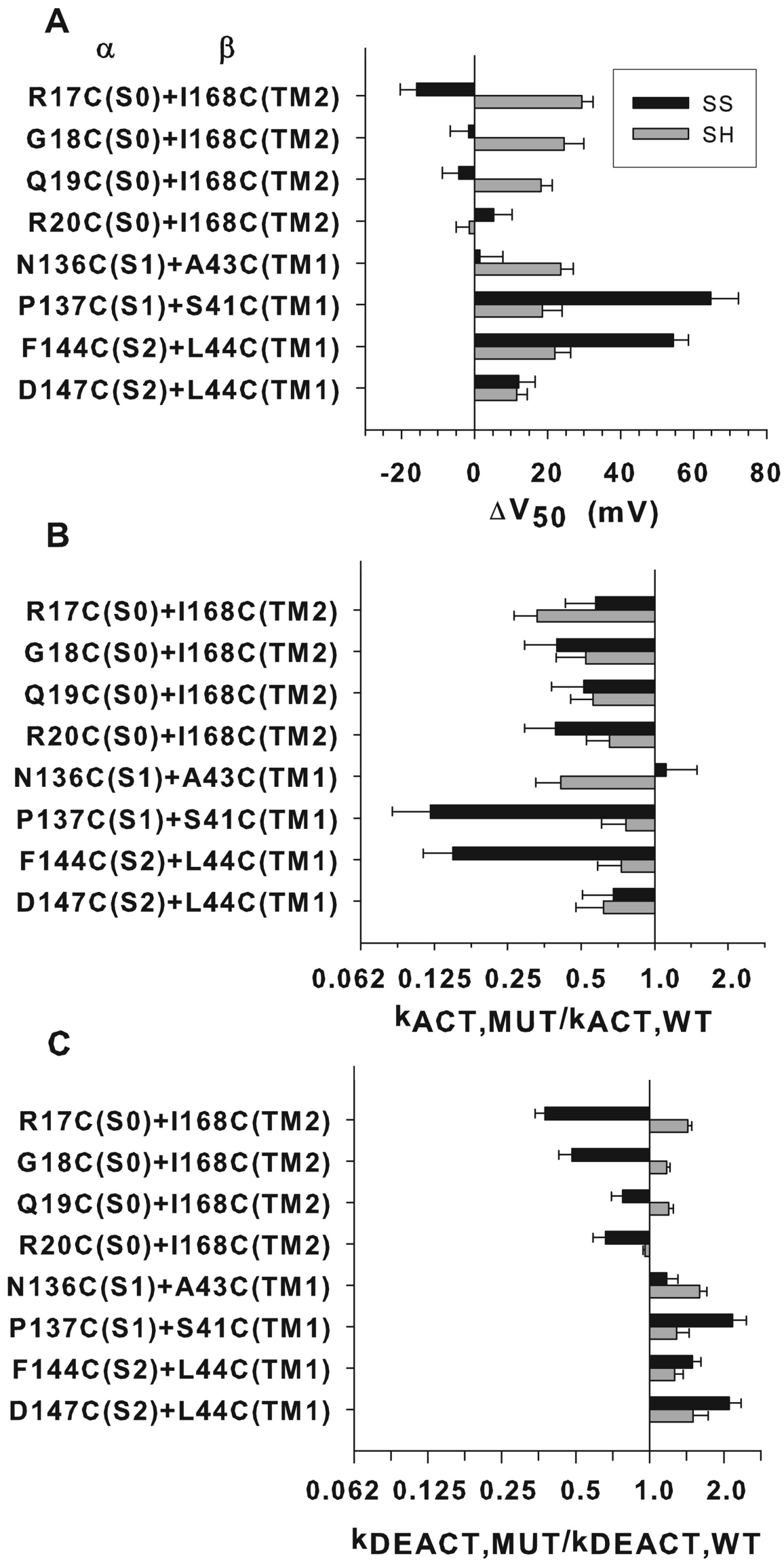

Functional effects of crosslinks

Eight of the most highly crosslinked pairs of Cys-substituted $\alpha$ and $\beta 4$ were tested for the functional effects of the double mutation to Cys and for the effects of crosslinking these Cys. In six of these eight double-mutant channels, neither the substitution of cysteines, per se, nor their disulfide crosslinking had more than a modest effect on function. It seems unlikely that the contacts between the flanking regions themselves are crucial for the modulation of $\alpha$ by $\beta 4$. It is quite likely that the underlying membrane-embedded helices, at least at their extracellular ends, are also in contact. Whether these contacts are essential in the modulation we do not yet know.

If there were extensive relative movement of these helices during activation, deactivation, or gating, crosslinking the extracellular flanks should affect one or more of these processes. If the relative movement were modest, it could be absorbed by the flexibility of the crosslinked flanks.

All four crosslinks S0 to $\beta 4$ TM2 crosslinks tested decreased the rate constants for both opening and closing but at most threefold (Fig. 6). For each crosslinked pair, the effects on opening and closing were of similar magnitude, consistent with the modest effect of each of the crosslinks on $V_{50}$ for opening. The crosslinks favored neither the open nor the closed state but somewhat retarded the transitions between them.

One of two crosslinks between S1 and $\beta 4$ TM1 and one of two between S2 and TM1 slowed opening eightfold and accelerated closing twofold. In these cases, the $V_{50}$ was shifted $\sim 60 \mathrm{mV}$ to the right. These crosslinks strongly favored the closed state. In the other two pairs, however, there was little effect of crosslinking. Thus, it is unlikely that TM1 either translates or rotates relative to $\mathrm{S} 1$ and $\mathrm{S} 2$ during opening or closing; rather, the marked effects of the first two crosslinks were likely attributable to a propagated perturbation of the structure.

Figure 6. Functional effects of the substitution of native residues by cysteines and their endogenous disulfide crosslinking. Eight pairs of Cys-substituted $\alpha$ and Cyssubstituted $\beta 4$ were expressed in HEK 293 cells. Recordings were in outside-out macropatches with $10 \mu \mathrm{M} \mathrm{Ca}{ }^{2+}$ in the pipette. Black bars show the effects attributable to the disulfide, and gray bars show the effects attributable to the substitution of the two Cys for native residues. Effects on $V_{50}(\boldsymbol{A})$, the rate constant for opening $(\boldsymbol{B})$, and the rate constant for closing $(C)$ were extrapolated to $100 \%$ crosslinking or $100 \%$ reduction as described previously (Liu et al., 2008b). SS, Disulfide-bonded Cys; SH, reduced Cys. 
Crosslinking of $\beta 4$ to $\alpha$ has, in general, less of an effect on $V_{50}$ than does crosslinking of $\beta 1$ to $\alpha$ (supplemental Fig. 4 , available at www.jneurosci.org as supplemental material), perhaps because there is less of an effect of $\beta 4$ on $V_{50}$ in the first place. Both $\beta 1$ and $\beta 4$ slow opening and closing of the channel. In both cases, TM2 is interacting with $\mathrm{S} 0$, which closely interacts with $\mathrm{S} 3-\mathrm{S} 4$, the movement of which is central to voltage-sensing and activation and deactivation. It is possible that, in both $\beta 1$ and $\beta 4$, this indirect interaction with $\mathrm{S} 3-\mathrm{S} 4$ retards both activation and deactivation of the voltage sensors. What subtle differences in the interactions of $\beta 1$ and $\beta 4$ lead the first to strongly favor the open state in the presence of $\mathrm{Ca}^{2+}$ and the second not to do so is a question for future study.

\section{References}

Becker L, Nesheim ME, Koschinsky ML (2006) Catalysis of covalent Lp(a) assembly: evidence for an extracellular enzyme activity that enhances disulfide bond formation. Biochemistry 45:9919-9928.

Behrens R, Nolting A, Reimann F, Schwarz M, Waldschütz R, Pongs O (2000) hKCNMB3 and hKCNMB4, cloning and characterization of two members of the large-conductance calcium-activated potassium channel beta subunit family. FEBS Lett 474:99-106.

Betzel C, Lange G, Pal GP, Wilson KS, Maelicke A, Saenger W (1991) The refined crystal structure of alpha-cobratoxin from Naja naja siamensis at 2.4-A resolution. J Biol Chem 266:21530-21536.

Brenner R, Jegla TJ, Wickenden A, Liu Y, Aldrich RW (2000) Cloning and functional characterization of novel large conductance calcium-activated potassium channel beta subunits, hKCNMB3 and hKCNMB4. J Biol Chem 275:6453-6461.

Brenner R, Chen QH, Vilaythong A, Toney GM, Noebels JL, Aldrich RW (2005) BK channel beta4 subunit reduces dentate gyrus excitability and protects against temporal lobe seizures. Nat Neurosci 8:1752-1759.

Cui J, Aldrich RW (2000) Allosteric linkage between voltage and $\mathrm{Ca}^{2+}$. dependent activation of BK-type mslol $\mathrm{K}^{+}$channels. Biochemistry 39:15612-15619.

Jiang XM, Fitzgerald M, Grant CM, Hogg PJ (1999) Redox control of exofacial protein thiols/disulfides by protein disulfide isomerase. J Biol Chem 274:2416-2423.

Jiang Y, Lee A, Chen J, Cadene M, Chait BT, MacKinnon R (2002) Crystal structure and mechanism of a calcium-gated potassium channel. Nature 417:515-522.

Jin P, Weiger TM, Levitan IB (2002) Reciprocal modulation between the alpha and beta 4 subunits of hSlo calcium-dependent potassium channels. J Biol Chem 277:43724-43729.

Kosower EM, Kosower NS, Kenety-Londner H, Levy L (1974) Glutathione. IX. New thiol-oxidizing agents: DIP, DIP +1 , DIP +2 . Biochem Biophys Res Commun 59:347-351.

Lee SY, Banerjee A, MacKinnon R (2009) Two separate interfaces between the voltage sensor and pore are required for the function of voltagedependent $\mathrm{K}^{+}$channels. PLoS Biol 7:e47.

Liu G, Zakharov SI, Yang L, Deng SX, Landry DW, Karlin A, Marx SO (2008a) Position and role of the BK channel alpha subunit S0 helix inferred from disulfide crosslinking. J Gen Physiol 131:537-548.

Liu G, Zakharov SI, Yang L, Wu RS, Deng SX, Landry DW, Karlin A, Marx SO (2008b) Locations of the betal transmembrane helices in the BK potassium channel. Proc Natl Acad Sci U S A 105:10727-10732.

Long SB, Campbell EB, Mackinnon R (2005) Voltage sensor of Kv1.2: structural basis of electromechanical coupling. Science 309:903-908.

Ma Z, Lou XJ, Horrigan FT (2006) Role of charged residues in the S1-S4 voltage sensor of BK channels. J Gen Physiol 127:309-328.

Meera P, Wallner M, Toro L (2000) A neuronal beta subunit (KCNMB4) makes the large conductance, voltage- and $\mathrm{Ca}^{2+}$-activated $\mathrm{K}^{+}$channel resistant to charybdotoxin and iberiotoxin. Proc Natl Acad Sci U S A 97:5562-5567.

Morrow JP, Zakharov SI, Liu G, Yang L, Sok AJ, Marx SO (2006) Defining the BK channel domains required for beta1-subunit modulation. Proc Natl Acad Sci U S A 103:5096-5101.

Nakatsukasa K, Brodsky JL (2008) The recognition and retrotranslocation of misfolded proteins from the endoplasmic reticulum. Traffic 9:861-870.

Nikles D, Tampé R (2007) Targeted degradation of ABC transporters in health and disease. J Bioenerg Biomembr 39:489-497.

Orio P, Rojas P, Ferreira G, Latorre R (2002) New disguises for an old channel: MaxiK channel beta-subunits. News Physiol Sci 17:156-161.

Wang B, Rothberg BS, Brenner R (2006) Mechanism of beta4 subunit modulation of BK channels. J Gen Physiol 127:449-465.

Wilkinson B, Gilbert HF (2004) Protein disulfide isomerase. Biochim Biophys Acta 1699:35-44.

Yarov-Yarovoy V, Baker D, Catterall WA (2006) Voltage sensor conformations in the open and closed states in ROSETTA structural models of $\mathrm{K}^{+}$ channels. Proc Natl Acad Sci U S A 103:7292-7297.

Zakharov SI, Morrow JP, Liu G, Yang L, Marx SO (2005) Activation of the BK (SLO1) potassium channel by mallotoxin. J Biol Chem 280:30882-30887.

Zhang X, Solaro CR, Lingle CJ (2001) Allosteric regulation of BK channel gating by $\mathrm{Ca}^{2+}$ and $\mathrm{Mg}^{2+}$ through a nonselective, low affinity divalent cation site. J Gen Physiol 118:607-636. 